4

\title{
Extensive exploration of Ayurvedic herbs to prioritize anti-viral drugs alike phytochemicals against SARS-CoV-2 using network pharmacology
}

\author{
Neha Choudhary, Vikram Singh ${ }^{*}$
}

Centre for Computational Biology and Bioinformatics, School of Life Sciences, Central University of Himachal Pradesh, Dharamshala, India -- 176206.

*Email: vikramsingh@cuhimachal.ac.in

\section{Abstract}

The novel coronavirus disease (COVID-19), which emerged in Wuhan, China, is continuously spreading worldwide, creating a huge burden on public health and economy. Currently, no specific vaccine or drug exists against SARS-CoV-2 virus, the causative agent of COVID-19. Ayurveda, the oldest healing-schema of Traditional Indian Medicinal (TIM) system, is considered as a promising CAM therapy to combat various diseases and disorders. To explore the regulatory mechanisms of 7,258 Ayurvedic herbs (AHs) against SARS-CoV-2, in this study, multi-targeting and synergistic actions of the constituent 34,472 phytochemicals (APCs) are investigated using a comprehensive approach comprising of network-pharmacology and molecular docking. By evaluating 292 APCs having high-level of similarity with anti-viral drugs in DrugBank for their binding affinity against 24 SARS-CoV-2 proteins, we develop and analyze a high confidence "Bi-regulatory network" of 115 APCs having ability to regulate protein targets in both virus and its host human-system. Immunomodulatory prospects of the antiviral drugs alike potentially effective phytochemicals (PEPs) are presented as a special case study, highlighting the importance of 6 AHs (Zea mays, Cucurbita maxima, Pisum sativum, Thlaspi arvense, Calophyllum inophyllum, Ziziphus jujuba) in eliciting the antiviral immunity at initial stages of infection. The mechanistic actions of PEPs against cardiovascular complications, diabetes mellitus and hypertension are also investigated to address the regulatory potential of Ayurvedic herbs in dealing with COVID-19 associated comorbidities. The study further reports 12 PEPs as promising source of COVID-19 comorbidity regulators.

Keywords: Ayurveda, COVID-19, SARS-CoV-2, Network pharmacology, Immunomodulators, Comorbidity, Anti-viral drugs. 


\section{Introduction}

In December 2019, a novel coronavirus caused an outbreak of pneumonia in Wuhan, Hubei province of $\mathrm{China}^{1}$, and since then it has rapidly transmitted across the world ${ }^{2}$ leading to the situation of Public Health Emergency of International Concern (PHEIC). The pathogen for leading coronavirus related pneumonia disease (COVID-19) has been classified as severe acute respiratory syndrome coronavirus 2 (SARS-CoV-2) by the International Committee on Taxonomy of Viruses. Compared to SARS-CoV responsible for the outbreak of SARS in 2003, the current risk of COVID-19 pandemic is mainly due to the high-transmission rate of SARSCoV-2. Currently, no specific or preventive treatment against SARS-CoV-2 infection is available. Based on the data from inspection-series or in-vitro experiments, few investigational agents have been suggested anecdotally, however, no specific drug has been approved by the US Food and Drug Administration (FDA) for COVID-19 till date. In the present scenario, infectioncontrol and preventive measures, including respiratory support through oxygen-therapy and mechanical ventilator (in severe cases), are the only methods being adopted for the clinical management of COVID-19.

The concept of drug-repurposing has become an attractive proposition for the identification of potentially active drugs against various diseases. With the time-consuming process, substantial costs and high failure rates of the development of new drugs, the reuse of existing drugs for other diseases offers an attractive schema for its lower developmental costs and shorter developmental timeline. The notion of drug repurposing is based on the multi-targeting ability of drugs which can be used to deal with various other diseases as disease pathogenesis is multi-factorial in nature $^{3}$. The concept has been used for past several years to repurpose existing drugs against various other diseases than the disease they have been originally developed for ${ }^{4,5}$. For COVID-19 also, the concept has been exploited to suggest potential existing drugs as there is an urgent requirement of drugs (single or combination based) to combat the disease. Recently, the antiviral drug repurposing approach have been implicated to a great extent to deal with SARS-CoV$2^{6}$

In response to the current demand for a suitable vaccine, the research community has jumped into the race to find a cure. To find an answer to that, China has turned its way towards traditional therapies by promoting TCM (Traditional Chinese Medicine) as a common 
prescription against COVID-19 $9^{7,8}$. The underlying mechanism lies in the multi-targeting nature of natural herbs, that in addition to providing strong immunity support, targets various ribosomal proteins, and thereby inhibiting the viral replication event ${ }^{7}$. Ministry of AYUSH, Government of India has also issued an advisory to use the Ayurveda, Siddha, Unani and Homeopathy as preventive measures ${ }^{9}$. Ayurveda, the traditional Indian knowledgebase of TIM system (Traditional Indian Medicine) which translates to "knowledge of life" is considered as the oldest healing schema originated more than four thousand years ago. Historical background of these medicines is also supporting the use of this system of medicine as preventive measures against variety of diseases and disorders including viral infection ${ }^{10}$. However, there is no controlled supporting data available for the use of any of these traditional medicines, and their efficacy for COVID-19 is unknown. Hence, the research scope of Ayurvedic medicines with valid scientific evidence is much worthy to combat the pandemic of COVID-19.

In recent years, a novel paradigm that integrates the concepts of network science and pharmacology, namely, network pharmacology has made its headway in the research of drug discovery and development ${ }^{11}$. The approach of network-pharmacology has proven to be a promising strategy towards next-generation approach of drug discovery for traditional medicines ${ }^{12,13}$. In this study, the information of Ayurvedic herbs was collected for their phytochemical composition and studied for their efficacies against COVID-19 using the approach of network pharmacology. A comprehensive dataset of phytochemicals was prepared for each herb using the information available at public domain databases. The therapeutic relevance of the phytochemicals was estimated using several protein target prediction algorithms. The prioritization of phytochemicals effective in managing COVID-19 was performed using the multi-step strategy involving similarity analysis with antiviral drugs, binding-affinity analysis against SARS-CoV-2 proteins, immune-regulatory potential, comorbidity analyses etc. We believe that the comprehensive methodology adopted in this study can serve as a powerful tool in deciphering the possible mechanism of action of Ayurvedic herbs of TIM origin for their management towards the global pandemic caused by novel coronavirus. Furthermore, the study may also serve as a universal guide towards illuminating the mechanisms of prescription of TIM against various other diseases and disorders. 


\section{Material and Methods}

\subsection{Dataset of Ayurvedic herbs:}

The information of the Ayurvedic herbs was collected from Indian medicinal plants database (IMPD) (http://www.medicinalplants.in/) which enlists the information of 7,258 unique herbs used in Indian medicinal system of Ayurveda as on March, 2018. The scientific names of the herbs available at IMPD can be checked in Supplementary Table-1.

\subsection{Phytochemical dataset of Ayurvedic herbs:}

A comprehensive list of the phytochemicals present in each Ayurvedic herb was developed using five database sources IMPPAT (Indian Medicinal Plants, Phytochemistry And Therapeutics) ${ }^{14}$, TCM-MeSH ${ }^{15}$, PCIDB (PhytoChemical Interactions DB) (https://www.genome.jp/db/pcidb), NPASS (Natural Product Activity and Species Source database) ${ }^{16}$ and Duke's phytochemical database (https://phytochem.nal.usda.gov/phytochem/search). For this, genus and species name of each herb was selected and inspected for their presence in the aforementioned databases. Out of 7,258 herbs in IMPD, we considered only those herbs in this study for which we could identify at least one phytochemical in the aforementioned databases. Two chemical databases, namely, PubChem ${ }^{17}$ and ChEMBL $^{18}$ were used for mapping the phytochemicals for their chemical information. The Ayurvedic phytochemicals (APCs) for which no chemical mapping could be obtained were not considered in this study. Following these steps, a dataset of 3,049 herbs and their varieties (in total, 3,966) was prepared and used in the further studies.

The hierarchical-chemical classification of APCs was performed using "Classyfire" which utilizes the chemical-ontology based information of 4,825 organic and inorganic compounds to predict the chemical class of query molecule ${ }^{19}$. For clustering of APCs, cluster services available at ChemMine tools were chosen ${ }^{20}$. The ChemMine-algorithm was used to calculate atom pair descriptors (i.e. features) of each subjected query compound. Using the set of unique and common features, a similarity matrix was constructed and the matrix was presented in the Newick tree format. The chemical information obtained from Classyfire server was added to the tree-format to display complete information associated with each APC molecule. The chemical classification was restricted to the APCs screened-in at the stage of "Anti-viral drug similarity calculations" (described in detail in the Material and Methods section 2.4). 
121 The information of human proteins targeted by APCs was compiled from STICH5.0, 122 SwissTargetPrediction and BindingDB. STITCH utilizes the information of manually curated as 123 well as experimental data for cataloguing chemical-target pairs ${ }^{21}$. For accessing high confidence 124 interaction pairs, the STITCH data was compiled at the confidence score of $\geq 0.4$. 125 SwissTargetPrediction is accessible through a web-based tool available at 126 http://www.swisstargetprediction.ch/ and offers predictions based on similarity principle through 127 reverse screening approach ${ }^{22}$. For each APC, only top-15 predictions from 128 SwissTargetPrediction were incorporated for the analysis. BindingDB is a web-accessible public 129 platform containing the binding information of about 7,493 proteins and 820,433 chemical 130 entities $^{23}$. The targets from BindingDB were screened corresponding to molecules having 131 chemical similarity $\geq 0.85$.

\subsection{Anti-viral drug dataset and similarity index calculation}

DrugBank database (https://www.drugbank.ca/) was used to collect the information of currently used anti-viral drugs (AVDs). Only, AVDs corresponding to the class of small-molecules were used in this study. For assessing the similarity between AVDs and APCs, a similarity measure based on Tanimoto coefficient $\left(T_{c}\right)$ was calculated for each pair of 34,472 APCs and 125 AVDs. For the calculation, the chemical structure of input molecule was encoded in form of binary digits using molecular fingerprints. A path-based molecular fingerprint, namely, FP2 which indexes the input molecule up to the length of seven atoms, was used for $T_{c}$ calculation using OpenBabel $^{24} . T_{c}$ between two chemical compounds A and B is given by

$$
T_{C_{(A, B)}}=\frac{N_{(A, B)}}{N_{(A)}+N_{(B)}-N_{(A, B)}}
$$

142 where, $N_{(A)}$ and $N_{(B)}$ represent the number of molecular fingerprints associated with chemical 143 compounds $\mathrm{A}$ and $\mathrm{B}$, respectively. The number of molecular fingerprints common to both the 144 chemical compounds is represented by $N_{(A, B)}{ }^{25}$. The value of the $T_{C(A, B)}$ ranges in between $0-1$, 145 with 0 representing no similarity and 1 representing maximum similarity between the 
compounds. The $T_{c}$ values between APCs and AVDs (obtained from DrugBank) are listed in Supplementary Table-2. The information of AVDs is also given in Supplementary Table-2.

To screen APCs capable of providing similar regulatory effects to existing anti-viral drugs, the two-condition based selection criterion was adopted ${ }^{26}$. Of these two conditions, first involves the selection of APCs whose Tc similarity is greater than 0.85 , and the second one includes the APCs whose $T c$ value $\neq 1$ and SMILES exactly similar against any of the 125 AVDs. Using this criterion, 292 APCs referred to as "potentially effective phytochemicals" (PEPs) could be identified.

\subsection{Disease association of the protein targets}

DisGeNET, a repository containing the information of gene-disease associations linked to Homo sapiens was used to investigate the association of protein targets into various disease classes ${ }^{27}$.

\subsection{In-silico molecular docking and interaction analysis}

Molecular docking and binding-energy (B.E.) calculations were used to assess the favorable conformation of ligand on to the protein active-site. The 3D-structures of 24 SARS-CoV-2 proteins were obtained from the I-TASSER platform available at https://zhanglab.ccmb.med.umich.edu/COVID-19/ and their molecular interaction with PEPs were studied using Autodock v4.2 $2^{28}$ and Autodock Vina packages ${ }^{29}$. The AutoDock combines the grid and simulated annealing-based algorithms to predict the best conformation of ligand inside the protein cavity. The B.E. values were calculated for each PEP molecules against the active site of each SARS-CoV-2 protein considered in the study. To screen and prioritize the list of PEPs against each protein of SARS-CoV-2, a screening cutoff was decided for each SARSCoV-2 protein on the basis of B.E. values distribution obtained from interactions with 292 PEPs. In order to select ligands with their best conformation inside the cavity of a SARS-CoV-2 protein, the high scoring SARS-CoV-2 protein - PEP pairs with B.E. values $<(\mu-\sigma)$ were considered for further studies, where $\mu$ is the mean of the 292 B.E. values and $\sigma$ is their standard deviation. In this manner, out of 292 PEPs, 129 were screened-in against 24 SARS-CoV-2 proteins and were refereed to as $\mathrm{PEP}_{\text {cov2 }}$ i.e. potentially effective phytochemicals against SARS- 
$175 \mathrm{CoV}-2$ proteins. The list of $129 \mathrm{PEP}_{\text {cov } 2}$ and their B.E. values with SARS-CoV-2 proteins is 176 given in Supplementray Table-3.

\section{Results and Discussion:}

\subsection{Phytochemical dataset of Ayurvedic herbs}

Of 7,258 botanical names of Ayurvedic herbs mentioned in the Indian medicinal plants database, the database, exhaustive mining from five databases could result in the compilation of 34,472 APCs (Ayurvedic phytochemicals). The Tanimoto-based similarity screening (as mentioned in Material and Methods section 2.4) of these APCs against anti-viral drugs resulted in the selection of 292 APCs referred to as PEPs, and the further study focuses on the detailed examination of these PEPs. The detailed description of these PEPs with their phytochemical ID and chemical identifier is listed in Supplementary Table-3.

When checked for the presence of these phytochemicals in the Ayurvedic herbs, 292 PEPs were found to be distributed among 558 herb varieties. The detailed mapping of PEPs onto their respective herb can be checked in Supplementary Table-4. The information was used as input to construct the Ayurvedic herb-phytochemical network (AH-PEPs network) with network size of 850 nodes (558 herbs + 292PEPs) and 1,685 edges (Figure-1). Examining the distribution of PEPs among 558 herbs helped us identify that AH_0303-v1 contributes maximally to the PEPs category with 35 of its phytochemicals. The Ayurvedic herb AH_0303-v1 corresponds to Artemisia annua and earlier reported studies on the herb shows that the plant possess antiviral activity against SARS-CoV ${ }^{30}$. The alcoholic extract of the plant was one of the most potent herbal medicines used against SARS-CoV in 2005. Based on its anti-viral properties, researchers across the globe are also trying to explore the effectiveness of this herb against novel coronavirus disease, COVID-19 $9^{31}$.In addition to AH_0303-v1, other Ayurvedic herbs enriched with PEPs are AH_3088-v1: Zingiber officinale, AH_0879-v1: Curcuma longa with 24 and 20 PEPs, respectively. Both of these are well-known Ayurvedic herbs for their immune-boosting capacity and are also been studied for their efficacies against exposed asymptomatic cases associated with COVID- $19^{32}$. 


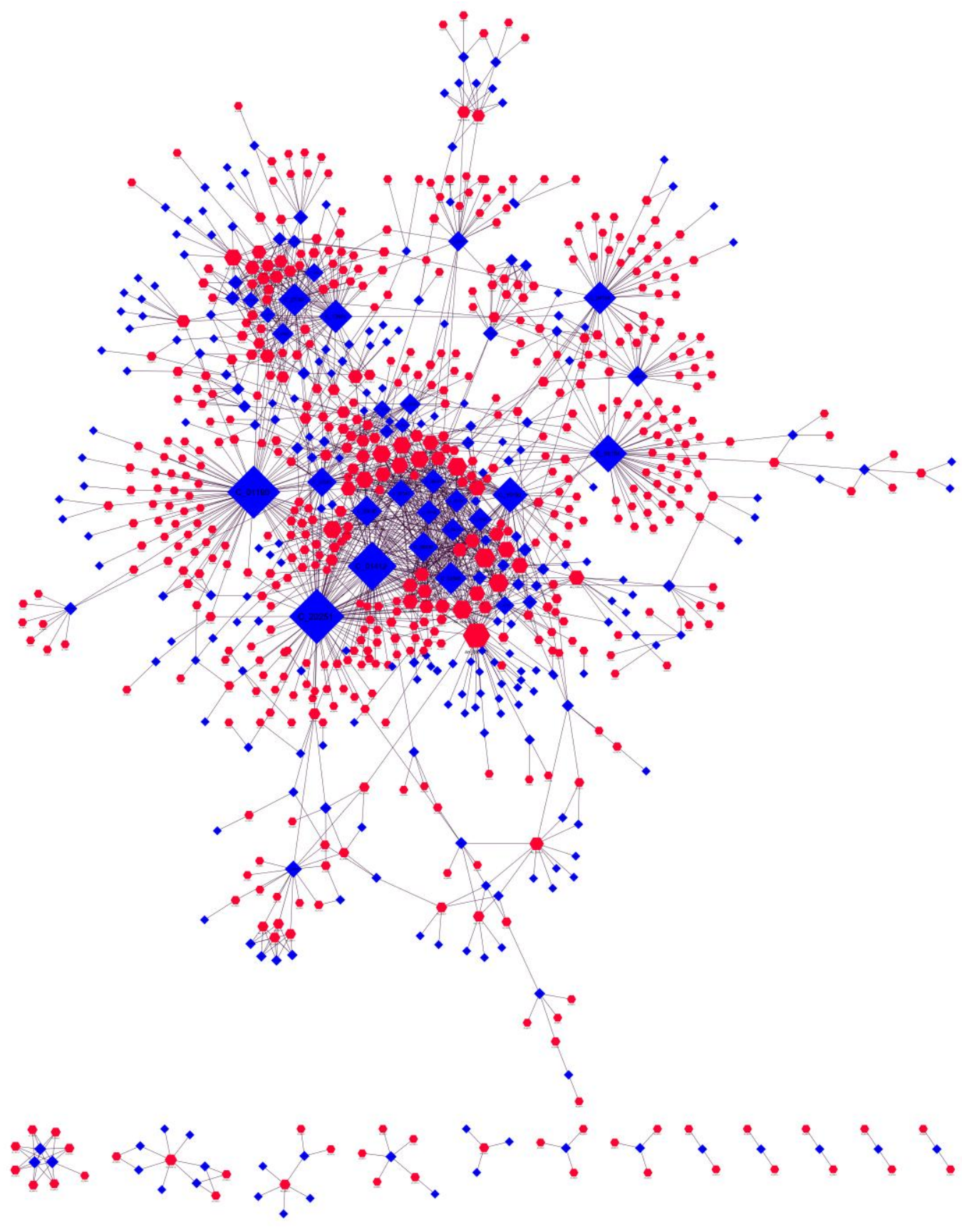

Figure 1. AH-PEP network: The AH-PEP network representing associations of 292 PEPs (blue colored triangles) with 558 herb varieties (red colored octagons). Herb AH_0303-v1 (Artemisia annua) contributes maximally to the PEPs category with 35 of its phytochemicals in the AH-PEP Network, as seen with largest node size in the network where the size of nodes varies according to its degree centrality.

The data suggests that the targeted action of these herbs against COVID-19 may be attributed to the constituting PEPs which hold the potential to regulate SARS-CoV-2 proteins (as may be seen 
211 in the analysis of $\mathrm{PEP}_{\text {cov2 } 2}-\mathrm{PT}_{\text {cov2 } 2}$ network, detailed in the later sections of this study). Detailed 212 examination of herbs may also put light on their respective phytochemicals for their target 213 specificity against SARS-CoV-2 proteins. The chemical organization of 292 PEPs was found to 214 be distributed among six broad chemical classes (Figure-2).

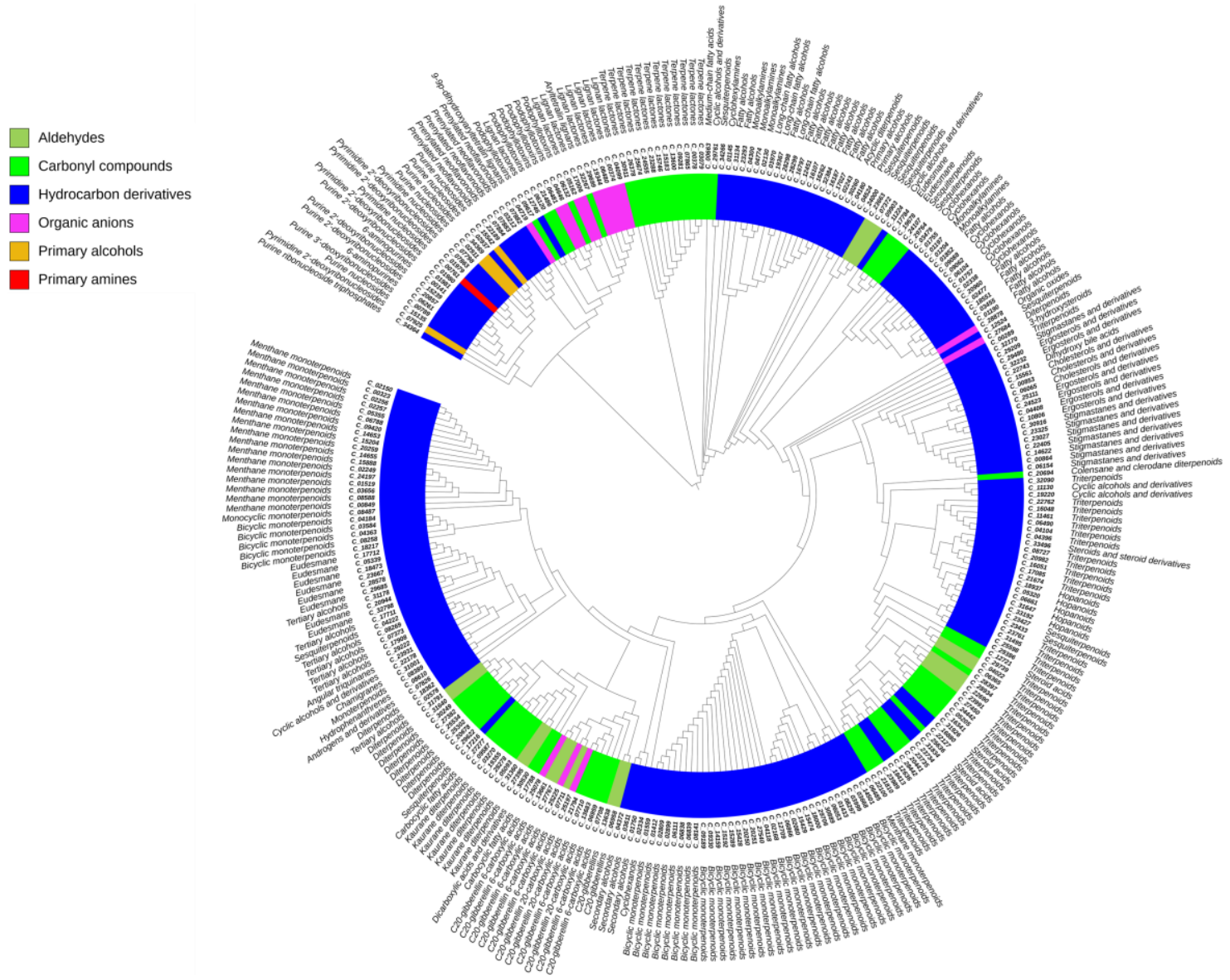

Figure 2. Clustering and chemical-distribution of PEPs: The 292 Potentially effective phytochemicals (PEPs) are clustered in a hierarchical-manner using Tanimoto-coefficient and atom-pair descriptors using ChemMine tools. Clustering of the PEPs is represented in the form of a tree-layout where outer circles represent the detailed-chemical class of PEP molecule and inner circle represents the PEP identifier assigned to each phytochemical considered in this study. The 292 PEPs are found to be broadly classified into 6 chemical classes and each class is represented by a unique color code.

225 Chemical mapping of the PEPs reveals that chemical classes of terpenoids especially 226 "Triterpenoids" and "Bicyclic monoterpenoids" were highly abundant in the dataset. This 
suggests that the PEPs dataset constitutes pharmaceutically relevant molecules as the class of terpenoids is of high importance in terms of pharmaceutical value due to their broad-spectrum medical application since prehistoric times ${ }^{33}$. Thus, future attention towards the detailed investigation of these PEPs could be of considerable importance in drug-discovery. The chemical class of each of the PEPs can be checked in Supplementary Tabel-5.

\subsection{Phytochemical-anti-viral drugs similarity network}

To select potentially active phytochemicals based on compound-compound similarity with existing anti-viral drugs, the Tanimoto-coefficients were calculated for each Ayurvedic phytochemical (APC) - anti-viral drug (AVD) pair. The similarity is depicted in the form of a bipartite-network, in which nodes in either set correspond to compounds from the lists of APCs or AVDs and edges are drawn between the nodes belonging to these two sets if the $T_{c}$ value between them follows the criterion mentioned in the Material and Methods section 2.4 (Figure3). Hence, only the APCs earlier passing the $T c$-based selection-criterion (referred as PEPs) were considered at this step. Satisfying this criterion, 292 PEPs were screened-in against 16 of 125 AVDs. In this manner, a $T_{c}$-based similarity-network between 292 PEPs and 16 AVDs, with network size of 307 nodes and 302 edges was constructed (referred as PEP-AVD similarity network; Figure-2). Detailed examination of the network returned that 160 PEPs share similarity with AV_DB00632 in the PEP-AVD network. AV_DB00632 corresponds to Docosanol, a class of approved drug effective against broad-spectrum lipid-enveloped viruses ${ }^{34}$. Among the list of 160 PEPs, C_00323 shares the maximum similarity with this AVD with $T c$ score of 0.92 . C_00323 is a cyclohexanol molecule that has gained massive attention for its isoprenylated forms, and is reported to be effective against viral infections as caused by HIV-1 and H1N $1^{35,36}$. According to the phytochemical-dataset prepared in this study, C_00323 is found to be present in 39 Ayurvedic herbs (including varieties), the abundance of this phytochemical in various Ayurvedic herbs strengthens the therapeutic relevance of Ayurveda against viral infections. 

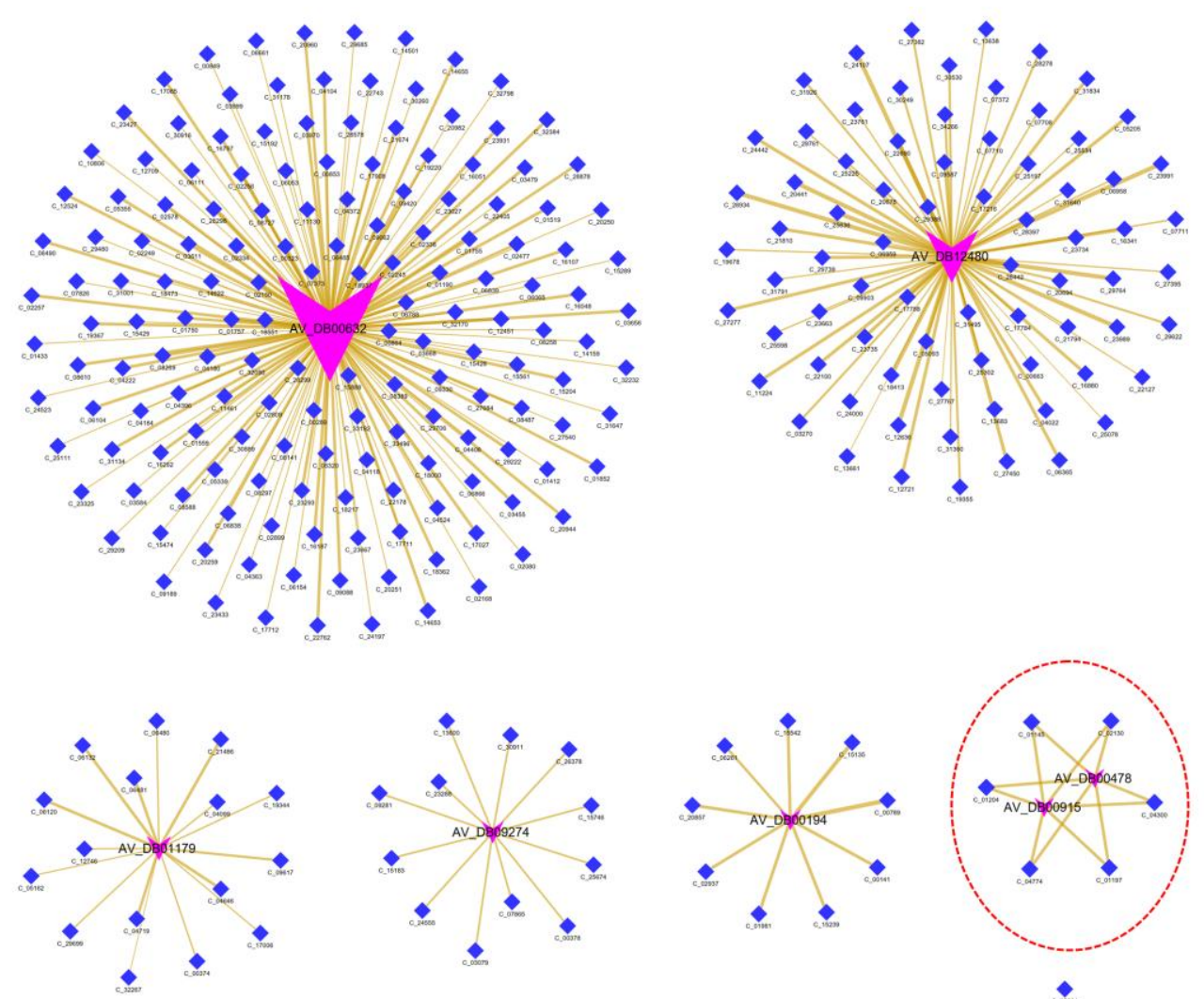

Figure 3. PEP-AVD similarity network: The PEP-AVD network represents the Tanimoto-coefficient $\left(T_{c}\right)$ based similarities between the PEPs and antiviral drugs listed in DrugBank. 292 PEPs (blue colored diamonds) are found to be associated with 16 (pink colored arrows) of total 125 AVDs considered in this study via 302 PEP-AVD pairs. Only PEP-AVD pairs following the selection criterion detailed in the Materials and Methods section are considered for constructing the network. The edge widths of 302 pairs in the network are plotted in proportion to their $T_{c}$ values. Red colored circular outlined sub-networks represent the multi-similarity APCs against more than one AVD class, highlighting 8 APCs (C_01204, C_04300, C_01145, C_02130, C_01197, C_07863, C_04774 and C_01979) having multi-level similarity. The size of the nodes varies according to its degree centrality value in this network.

It is interesting to note that while the majority of PEPs share one-to-one connection i.e. showing similarity with only one AVD, few of them have one-to-many similarity-based connections. Out of 292 PEPS, 8 (C_01204, C_04300, C_01145, C_02130, C_01197, C_07863, C_04774 and 
C_01979) were found to have similarity with more than one AVD, suggesting the importance of detailed examination of these compounds to be examined in detail for their molecular features thereby aiding in future pharmacophore-based anti-viral drug-design approaches.

\subsection{Phytochemicals -- SARS-CoV-2 protein target association}

As per the approach mentioned in Material and Methods section 2.6, each SARS-CoV-2 protein was associated with their screened-in PEPs and their association was represented in form of $\mathrm{PEP}_{c o v 2}-\mathrm{PT}_{\text {cov2 }}$ network (Figure-4). As already stated, the cutoff criterion resulted in selecting 129 of 292 PEPs against 24 SARS-CoV-2 proteins, therefore $\mathrm{PEP}_{\text {cov2 }}-\mathrm{PT}_{\text {cov2 }}$ network was limited to 153 nodes (129 $\mathrm{PEP}_{\text {cov } 2} \& 24 \mathrm{SARS}-\mathrm{CoV}-2$ proteins) having 1,179 edges between them. The information of $\mathrm{PEP}_{c o v 2}-\mathrm{PT}_{c o v 2}$ network can be checked in Supplementary Table-3.

For QHD43415_6, a non-structural protein nsp6 of SARS-CoV-2, 62 PEP $_{\text {cov2 }}$ were screened-in, where the least-binding energy was observed as $-8.3 \mathrm{kcal} / \mathrm{mol}$ for C_04396 and C_16048. Studies suggest that nsp6 is linked to the virulence of the virus as it is involved in the cellular DNA synthesis ${ }^{37,38}$. Similarly, for the main protease protein, QHD43415_3, $44 \mathrm{PEP}_{\text {cov2 } 2}$ were screened-in of which 4 (C_32090, C_11130, C_17085, and C_22189) show very good binding affinities with the lowest one being $-8.5 \mathrm{kcal} / \mathrm{mol}$. QHD43415_3 is a coronavirus 3 chymotrypsin-like protease (3CLpro) which is often termed as "the Achilles" heel of coronaviruses and is a validated target for identification of novel leads against corona virus ${ }^{39}$. Thus, the relevance of above mentioned 4 compounds in the regulation of QHD43415_3 is highly noticeable and requires special attention for in-vitro and in-vivo evaluation of their activity as potential anti-coronavirus inhibitors.

During the detailed analysis of local network structures, it was found that C_03212 possesses the multitargeting ability against 20 of 24 SARS-CoV-2 proteins. The shift from single-target to multi-target drugs has made rapid and remarkable progress and has emerged as an evolving paradigm of drug-discovery ${ }^{40}$, and as highlighted in a recent study network-pharmacology acts as a powerful tool in identifying effective combination therapies in drug development ${ }^{41}$. Hence, other protein targets may also be looked for their potential regulators from the $\mathrm{PEP}_{\text {cov } 2}-\mathrm{PT}_{\text {cov } 2}$ 
network and may be ranked on the basis of their binding energy values, thereby giving an overall idea about the protein-specific regulatory role of Ayurvedic herbs against COVID-19 disease.

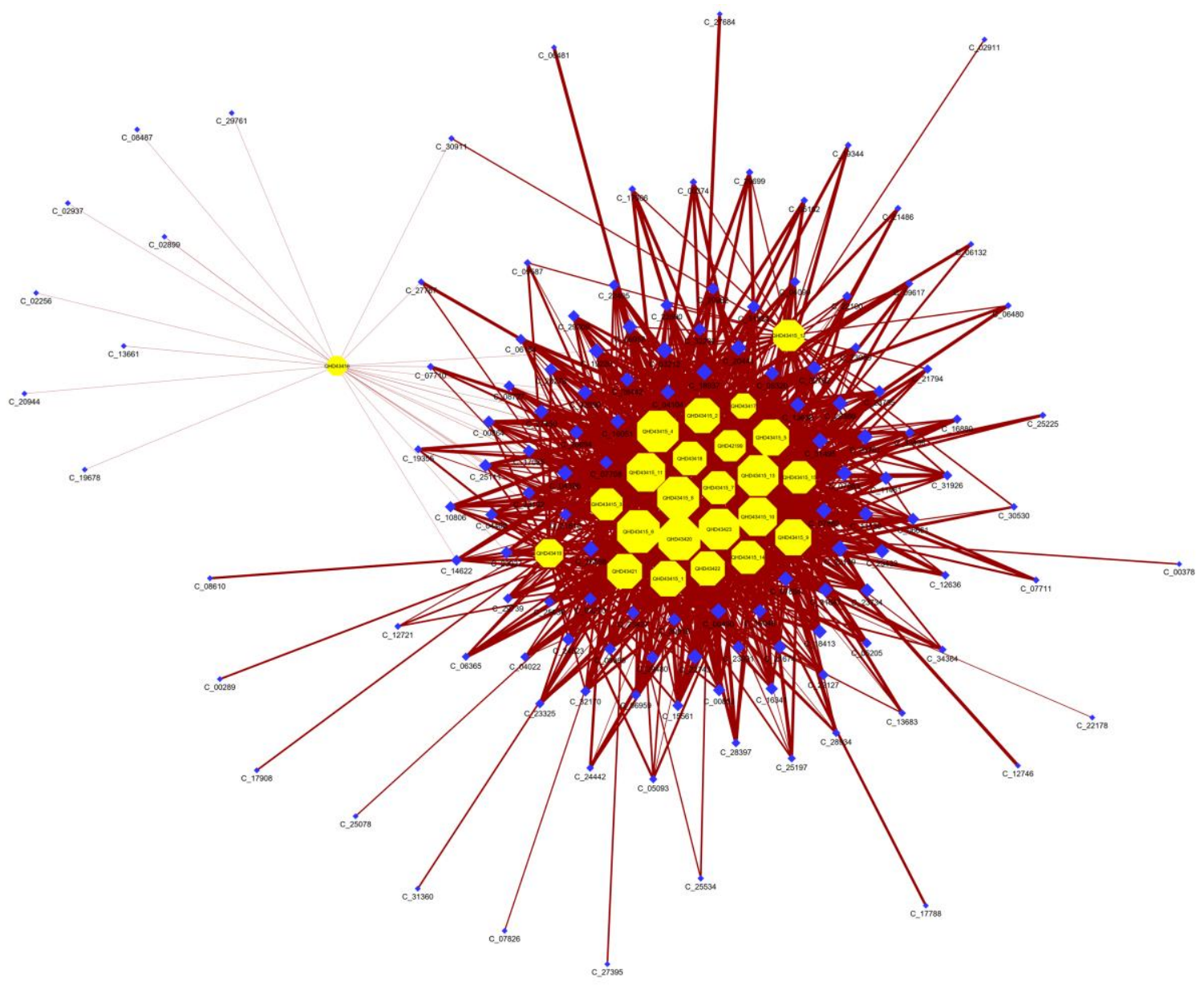

Figure 4. Phytochemicals--SARS-CoV-2 protein target association $\left(\mathbf{P E P}_{c o v 2}-\mathbf{P T}_{\text {cov2 }}\right.$ network): The $\mathrm{PEP}_{\text {cov2 }}{ }^{-}$ $\mathrm{PT}_{c o v 2}$ network represents the association of $129 \mathrm{PEP}_{c o v 2}$ with SARS-CoV-2 proteins leading to the network size of 153 nodes (129 $\mathrm{PEP}_{\text {cov } 2}$ and $24 \mathrm{SARS}-\mathrm{CoV}-2$ proteins) and 1,179 edges. The $\mathrm{PEP}_{\text {cov2 } 2}$ are represented using blue colored diamond shaped nodes and SARS-CoV-2 proteins $\left(\mathrm{PT}_{\text {cov2 }}\right)$ as yellow colored octagons. The size of the nodes varies according to its degree centrality and width of the edges varies according to their binding energy values, where the pairs having lower value of binding energy (which represents the most suitable protein-ligand interaction pair) are given more weight and are ranked higher.

\subsection{Phytochemicals-Human protein target association}

To detect the poly-pharmacological action of PEPs on the human system, the association of PEPs with their human PTs was represented in the form of Potentially Effective PhytochemicalsHuman Protein Target (PEPs-PT ${ }_{h s}$ ) network. For a phytochemical having ID C_31134, no 
protein target could be screened-in against the selection criterion adopted for protein target identification, therefore, the network was constructed by associating 291 PEPs with their 621 protein targets identified using three target prediction algorithms as mentioned in Material and Methods section 2.3. This resulted in the construction of PEPs-PT ${ }_{h s}$ network with network size of 912nodes (291 PEPs $+621 \mathrm{PT}_{h s}$ ) and 6,299 edges (Supplementary Figure-1). Each of the 6,299 $\mathrm{PEP}-\mathrm{PT}_{h s}$ interaction pairs was prioritized based on their prediction supported from three target prediction algorithms. This led to the identification of 1,265 high confidence pairs, as predicted by at least two of the three target prediction algorithms (HCI pairs). The information of the 6,299 PEP-PT $_{h s}$ pairs and the pairs corresponding to HCI data is detailed in Supplementary Table-6.

A sub-network of the PEP-PT ${ }_{h s}$ network consisting of 502 nodes and 2,690 edges, specific to 129 $\mathrm{PEP}_{c o v 2}$ and their $373 \mathrm{PTs}$ (referred to as $\mathrm{PEP}_{c o v 2}-\mathrm{PT}_{h s}$ network)was derived to focus on human proteins being targeted by them (Figure-5). In the $\mathrm{PEP}_{\text {cov2 }}-\mathrm{PT}_{h s}$ network, C_00289 and C_02937 hold the maximum targeting capacity among other $\mathrm{PEP}_{\text {cov2 }}$, as these can target 74 and 49 proteins, respectively. Their high degree centrality value represents the importance of these phytochemicals in the overall $\mathrm{PEP}_{c o v 2}-\mathrm{PT}_{h s}$ network. It was interesting to note that all the 129 $\mathrm{PEP}_{\text {cov2 }}$ were of multi-targeting nature with the capability to regulate several human proteins simultaneously. Among the protein targets, maximum number of regulators could be identified for Q96RI1, P28845 and P10275 with 102, 100 and $95 \mathrm{PEP}_{\text {cov2 }}$, respectively. P10275 is an androgen receptor encoded by AR gene and the relevance of the androgens has been associated with increased viral load and dissemination as observed in case of COVID ${ }^{42}$. Androgen-mediated induction of COVID-19 suggests that the role of these $95 \mathrm{PEP}_{\text {cov2 }}$ in regulating the AR gene is noticeable for the management of COVID-19.

Case-Study I: $\mathrm{PEP}_{\operatorname{cov} 2}$ as bi-directional regulators effective against COVID comorbidities.

Since the focus of the study is to identify phytochemicals with a regulatory role in both the pathogen and its host, bi-directional regulators were searched among the $\operatorname{PEP}_{\text {cov2 }}$ list. Such compounds have an added advantage as they work on dual scale mode, where at one end they can target pathogen proteins that may be crucial for its survival while at another end they tend to regulate the human proteins required to strengthen its defense mechanism against the pathogen. To identify the desired $\mathrm{PEP}_{c o v 2}$ with bi-directional regulation ability, $129 \mathrm{PEP}_{c o v 2}$ were examined 
against SARS-CoV-2 and human protein targets in the $\mathrm{PEP}_{\text {cov } 2}-\mathrm{PT}_{\text {cov2 } 2}$ and $\mathrm{PEP}_{\text {cov2 } 2}-\mathrm{PT}_{h s}$ networks, respectively.

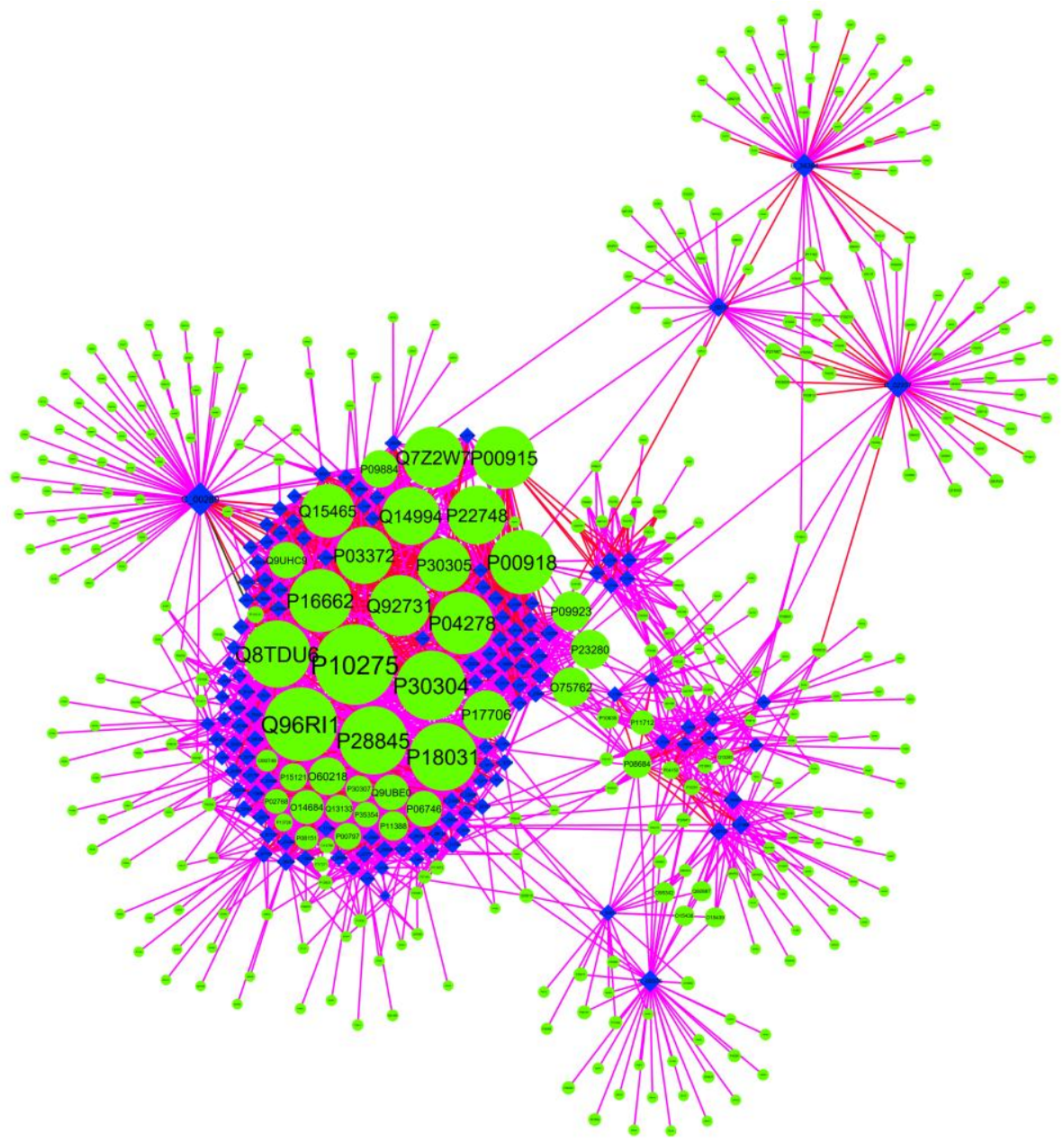

Figure 5. PEP cov2 $_{2}-\mathrm{PT}_{h s}$ network: $\mathrm{PEP}_{c o v 2}-\mathrm{PT}_{h s}$ network represents a sub-network of PEPs-PT $\mathrm{T}_{h s}$ network, specific to the association of $129 \mathrm{PEP}_{c o v 2}$ and their 373 human protein targets. The network consists of 502 nodes and 2,690 edges, with the size of nodes varying as per their degree values in the PEPs-PT ${ }_{h s}$ network.

In $\mathrm{PEP}_{\text {cov2 }}-\mathrm{PT}_{h s}$ network, $129 \mathrm{PEP}_{\text {cov } 2}$ were found to be associated with 373 human protein targets. Using all these data, a tripartite network consisting of $129 \mathrm{PEP}_{\text {cov2 }}$, their 373 human targets and 24 SARS-CoV-2 protein targets referred to as "Bi-regulatory $\mathrm{PEP}_{\text {cov2 }}$ network" was developed. The obtained network of size 526 nodes and 3,869 edges is given in Supplementary Figure-2; Supplementary Table-7. 

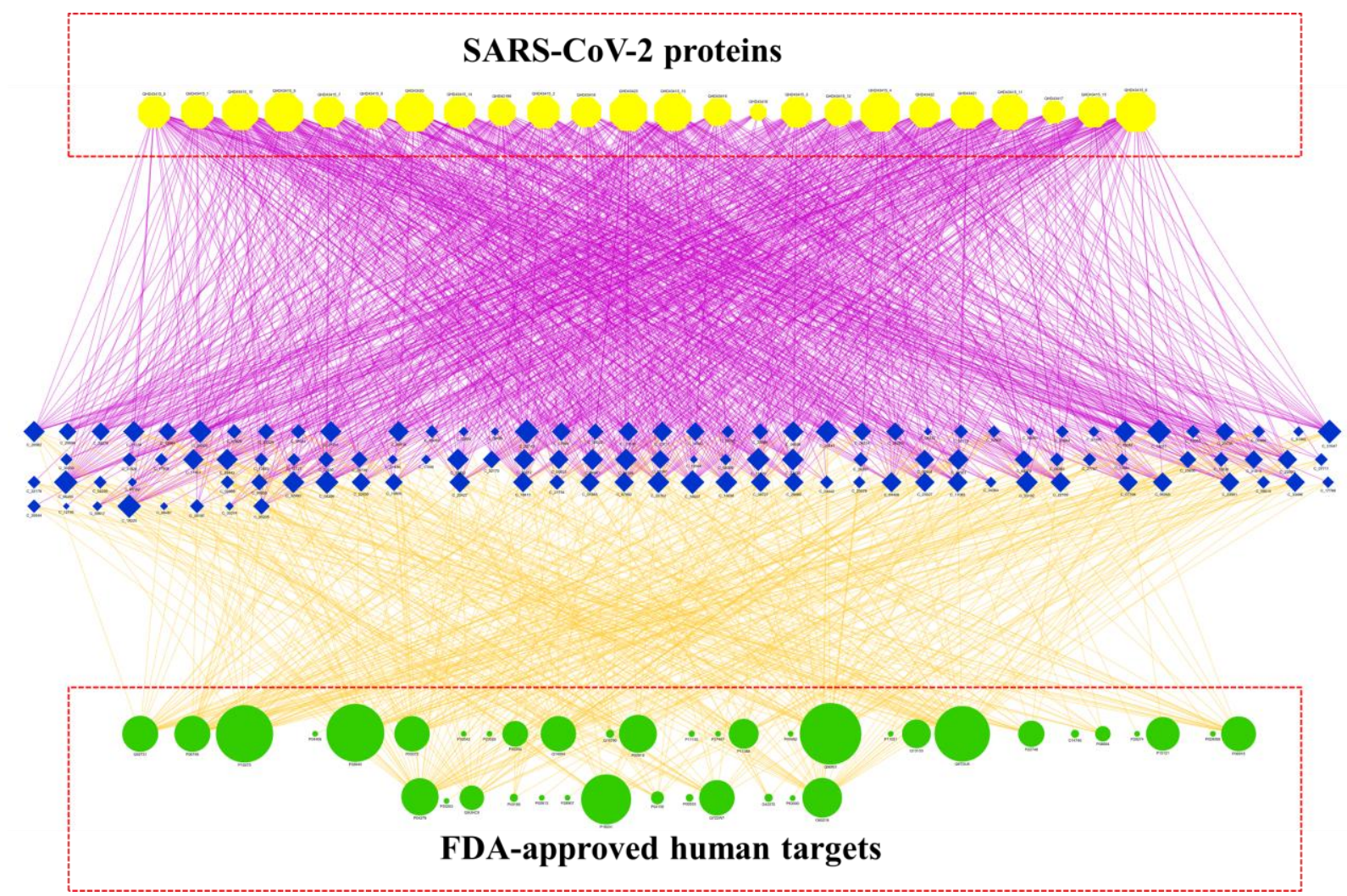

Figure 6. Druggable bi-regulatory $\mathbf{P E P}_{\text {cov2 }}$ network: The network represents the dual-regulatory mode of 115 $\mathrm{PEP}_{\text {cov2 }}$ (middle layer, blue diamond shaped nodes) against 24 SARS-CoV-2 proteins (top-layer, yellow-colored octagon nodes) and 40 approved protein targets of Homo sapiens (bottom-layer, green colored circular nodes). For the differentiation, the edges between $\mathrm{PEP}_{\text {cov } 2}$ and SARS-CoV-2 proteins are represented using violet color while edges between $\mathrm{PEP}_{c o v 2}$ and human-proteins using orange color. The size of the nodes among the network varies according to its degree in this network.

It is well known that not all proteins of the human system are suitable for drug-interactions, only a fraction of the total human proteome can bind to drug molecules with high affinity and are potential drug-targets, i.e. they have an association with a disease or disorder. Therefore, a "Druggable bi-regulatory $\mathrm{PEP}_{\text {cov } 2}$ network" was extracted from the "Bi-regulatory $\mathrm{PEP}_{\text {cov2 }}$ network" by considering only those proteins that have been approved by FDA to be studied as

366 drug targets. While "Bi-regulatory $\mathrm{PEP}_{\text {cov2 }}$ network" gives an overall idea of the dual-regulatory mode of $\mathrm{PEP}_{\text {cov } 2}$, the sub-network may provide valuable help in protein-specific drug-designing of $\mathrm{PEP}_{\text {cov2 }}$ with multi-targeting action. Confidence was also added at this level by considering only those $\mathrm{PEP}_{\text {cov2 }}-\mathrm{PT}_{h s}$ pairs that belong to the $\mathrm{HCI}$ data. In this manner, a high-confidence 
370 druggable-subnetwork of size 179 nodes and 2,250 edges, consisting of 24 SARS-CoV-2 371 proteins, $115 \mathrm{PEP}_{\operatorname{cov} 2}$ and 40 human-protein approved targets (Figure-6).

372 Recent studies on the treatment procedure given to COVID-19 patients address the need of 373 special attention towards the problem of cardiovascular system ${ }^{43}$. Since most of the current anti374 viral drugs cause cardiac complications, alternative therapeutic strategies effective to combat the 375 cardiac toxicity should be given consideration. Therefore, we searched for $\mathrm{PEP}_{\text {cov2 }}$ that can target 376 COVID-19 proteins without imposing a load on the cardiac system. To achieve the desired list of $377 \mathrm{PEP}_{\text {cov2 }}$, their protein targets in the human-system were checked for the participation in 378 cardiovascular diseases. To extract the high confidence disease association data, the Gene379 disease association (GDA) score $(S)$ of 0.05 was chosen as threshold ${ }^{44}$ so as to have a non-zero 380 contribution from either of the C (curated data), M (animal model data) or I (inferred data), or a 381 support of at least 5 publications. Thirty-six proteins among the 373 human targets of 129 $382 \mathrm{PEP}_{\operatorname{cov} 2}$, were found to be involved in cardiovascular diseases within the desired cut-off score. 383 The interactors specific to these 36 proteins were extracted from the Bi-regulatory PEP cov2 384 network, where they were found to have an association with $123 \mathrm{PEP}_{\text {cov } 2}$ and all the 24 SARS385 CoV-2 proteins and presented as a sub-network specific to cardiovascular diseases with network 386 size of 183 nodes and 1,471 edges (Supplementary Figure-3). In the network, the most 387 multitargeting $\mathrm{PEP}_{\text {cov } 2}$; $\mathrm{C}_{-} 03212$ shows its targeting action against 20 SARS-CoV-2 proteins and 388 also support the cardiac system by regulating 3 cardiovascular-diseases associated proteins i.e. 389 O00206 (TLR4), Q13093 (PLA2G7) \& P42336 (PIK3CA). C_03212 (Inophyllum B) 390 corresponds to the most active component of Calophyllum inophyllum, an important component 391 of Ayurvedic drug therapy. Besides regulating an important therapeutic target TLR4 ${ }^{45}$, $392 \mathrm{PLA} 2 \mathrm{G} 7^{46}$ and PIK3CA ${ }^{47}$ against various cardiac-related diseases, literature data is plenteous for 393 the anti-viral activity of C_03212 48,49 . These findings suggest that future research endeavors 394 towards exploring the anti-COVID-19 activity of C_03212 must be given a proper consideration 395 and examined in detail as per the in-vivo and in-vitro studies. 
Cardiovascular Diseases (36)

A.

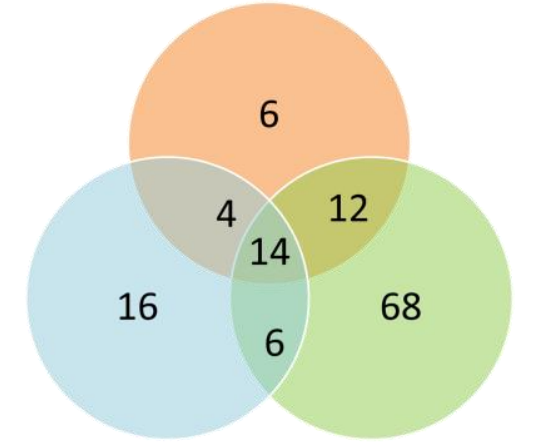

Hypertension (40) Diabetes (100)
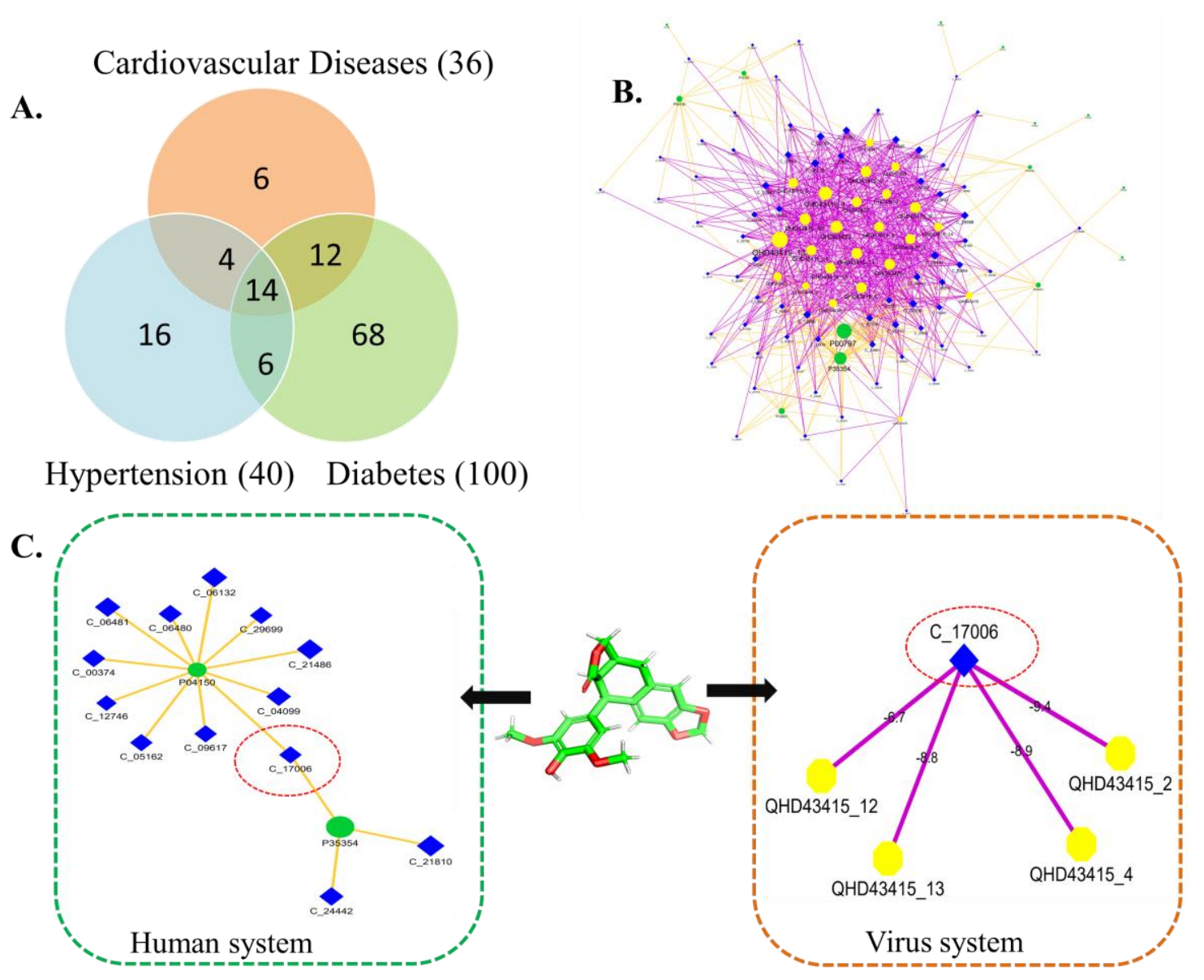

Figure 7. Regulatory role of phytochemicals in dealing COVID-19 associated comorbidities. A. Distribution of proteins among COVID-19 comorbid diseases: The Venn-diagram shows the distribution of human-protein targets of PEP cov2 $_{2}$ in 3 COVID-19 associated comorbid diseases, namely, cardiovascular diseases, hypertension and diabetes. Of total 373 Human targets of $129 \mathrm{PEP}_{\text {cov } 2}$ considered in this study, 36 were associated with cardiovascular diseases, 40 with hypertension and 100 with diabetes mellitus. 14 overlapping proteins common to all the 3comorbid diseases were identified and considered for detailed analysis. B. Bi-regulatory PEP $\mathbf{P}_{\boldsymbol{c o v}}$ network specific to 14 common proteins: The network is a subnetwork of Bi-regulatory $\mathrm{PEP}_{c o v 2}$ network specific to the $\mathrm{PEP}_{c o v 2}$ effective in dealing COVID-19 associated comorbidity diseases; cardiovascular diseases hypertension, and diabetes mellitus. The network contains 14 human-proteins (green colored circular nodes) being regulated by $73 \mathrm{PEP}_{\text {cov2 }}$. The information of SARS-CoV-2 proteins targeted by these $73 \mathrm{PEP}_{\text {cov } 2}$ are also added to the network. For the differentiation, the edges between $\mathrm{PEP}_{\text {cov } 2}$ and SARS-CoV-2 proteins are represented using violet color while edges between $\mathrm{PEP}_{\text {cov } 2}$ and human-proteins using orange color. The size of the nodes among the network varies according to their degree value, representing the high number of regulators for P04150 and P35354 (as depicted by their large size among all the nodes). C. Multi-targeting role of a bi-regulatory phytochemical C_17006: A phytochemical having ID C_17006 shows a dual action mode in both the human and SARS-CoV-2 systems. The multi-targeting nature of this compound against two human FDA-approved protein targets P04150 and P35354 is shown in the left side of the panel. In the virus system, the compound can target 4 viral proteins within the binding energy range of 9.4 to $-6.7 \mathrm{kcal} / \mathrm{mol}$ where best of $-9.4 \mathrm{kcal} / \mathrm{mol}$ was obtained for nsp2 protein QHD43415_2 (shown in the right side of the panel). The binding energy values of the compound with each viral protein are represented along the edges of the network. 
In the context of disease-comorbidities associated with COVID, a study by Roth and group suggests that the severity of COVID infection is higher for the patients suffering from diabetes mellitus and hypertension ${ }^{50}$. Therefore, using a similar strategy as applied for cardiovascular diseases, drug targets involved in diabetes and hypertension were also checked and a separate network for each disease was constructed and investigated. This is essential to analyze the underlying disease-comorbidity pattern and the compounds from Ayurvedic herbs that may regulate them while dealing against the COVID-19 infection. The proteins associated with each disease considered can be checked in Supplementary Table-8. For the identification of PEP cov2 $_{2}$ that may act on multiple scales, the protein targets of $\mathrm{PEP}_{\operatorname{cov} 2}$ were checked for their multidisease association, considering the above 3 comorbid diseases. Detailed examination could help us to identify that multi-disease associations of a protein were observed at this point, where multiple proteins overlap between the 3 classes of diseases discussed here (Figure-7.A).

The 14 human proteins common to all these 3 comorbid diseases (P35354, P02649, P00797, P37231, P04035, P08253, P16581, P30556, P04150, P35228, P42336, P12821, P06858, P29474) were selected and their regulatory $\mathrm{PEP}_{\text {cov2 }}$ were checked. The proteins specific to each disease class as well as those overlapping are given in (Supplementary Table-8). Mapping of these 14 proteins onto Bi-regulatory $P E P_{\text {cov2 }}$ network could help us in deriving a sub-network specific to them with size of 111 nodes (73 PEP cov2 $_{+} 14$ Human proteins +24 SARS-CoV-2 proteins) and 749 edges (Figure-7.B). To identify high-confidence regulatory $\mathrm{PEP}_{\text {cov2 }}$, only pairs corresponding to HCI-pair data were considered, leading to the selection of $12 \mathrm{PEP}_{\text {cov2 }}$ against 2 proteins (P04150 and P35354). Both these proteins i.e. P04150 and P35354 also belong to the FDA-approved protein target list, thereby suggesting the key relevance of these proteins targets and phytochemicals against COVID-19. Detailed association of these interactions helped us to highlight the multitargeting role of C_17006, as it targets both these protein targets (Figure-7.C). When checked for its SARS-CoV-2targeting capacity, C_17006 was found to have its binding affinity against 4 SARS-CoV-2 proteins within the binding energy range of -9.4 to $-6.7 \mathrm{kcal} / \mathrm{mol}$ where best of $-9.4 \mathrm{kcal} / \mathrm{mol}$ was noted for QHD43415_2, a non-structural protein 2 (nsp2), shown in Figure-7.C. This suggests the role of C_17006 is highly noteworthy in dealing with the co-morbidities associated with COVID-19. In this manner, other regulatory molecules can also be checked for their multi-targeting capacity and can be prioritized based on their binding affinity with SARS-CoV-2 proteins. 


\section{Case Study II: Immunoregulatory potential of PEP cov2}

450 To explore the underlying mechanisms of Ayurvedic herbs being studied towards promoting the 451 human immune system, a sub-network of immune pathways being regulated by PEP $c o v$ was 452 constructed. It has been studied that in the early stages of infection or during incubation period, 453 host needs a specific adaptive immune response to exterminate virus from the system ${ }^{51}$. This is 454 necessary to inhibit the progression of the disease to its chronic form or more severe stages. At 455 this stage immune-system of the host-body plays an important role to promote a state of good 456 health. A strong immune system in addition to the genetic background (e.g. HLA) is essential to 457 elicit a strong antiviral immunity at initial stages ${ }^{51}$. Therefore, immunomodulatory potential of $458 \mathrm{PEP}_{\text {cov2 }}$ was investigated by characterizing potential $\mathrm{PEP}_{\text {cov2 }}-\mathrm{PT}_{h s}$ interactions potentially 459 responsible for immune system pathways. For this, 21 pathways specific to immune-system as 460 described by KEGG database (i.e. hsa04062, hsa04610, hsa04611, hsa04612, hsa04620, 461 hsa04621, hsa04622, hsa04623, hsa04624, hsa04625, hsa04640, hsa04650, hsa04657, hsa04658, 462 hsa04659, hsa04660, hsa04662, hsa04664, hsa04666, hsa04670 \& hsa04672) and their protein 463 targets in human were selected for further analysis. Among 373 human protein targets of 129 $464 \mathrm{PEP}_{\text {cov2 }}$, 63 were found to be involved in the above mentioned 19 of 21 immune pathways via 465163 interactions (as presented in immunoregulatory network; Figure-8.A; Supplementary 466 Table-9). For two immune pathways hsa04624 and hsa04625, no protein target shows their 467 involvement and therefore network is restricted to 19 immune pathways. The network suggests 468 that the immunomodulatory potential of $\mathrm{PEP}_{\operatorname{cov} 2}$ is largely via regulating chemokine and NOD469 like receptor signaling pathways. The high regulatory potential of $21 \mathrm{PEP}_{\operatorname{cov} 2}$ against chemokine 470 signaling pathways may also give an added advantage in managing cardiovascular diseases like 471 atherosclerosis, as such patients are at high risk in developing COVID-19 infection ${ }^{43,52}$. The 472 chemokine regulation is mainly via these $21 \mathrm{PEP}_{\text {cov2 }}$ which target 18 proteins involved in this 473 pathway. The location of these 18 proteins onto the pathway is shown in red rectangles in $474 \quad$ Figure-8.B.

475 
A.

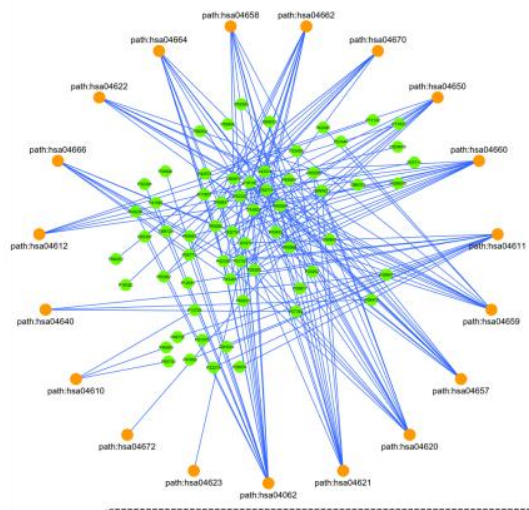

C.
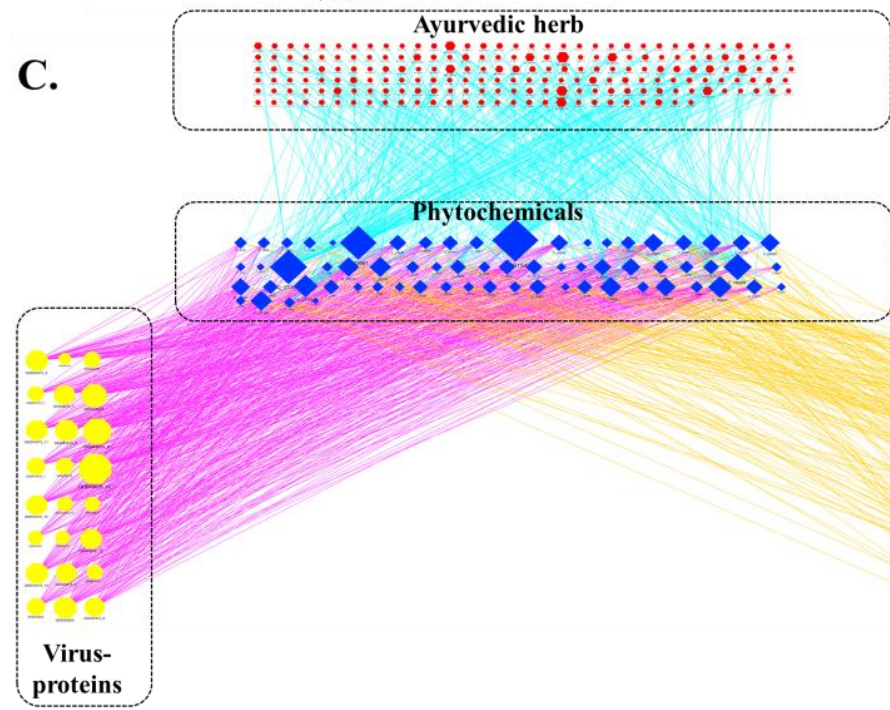

B.

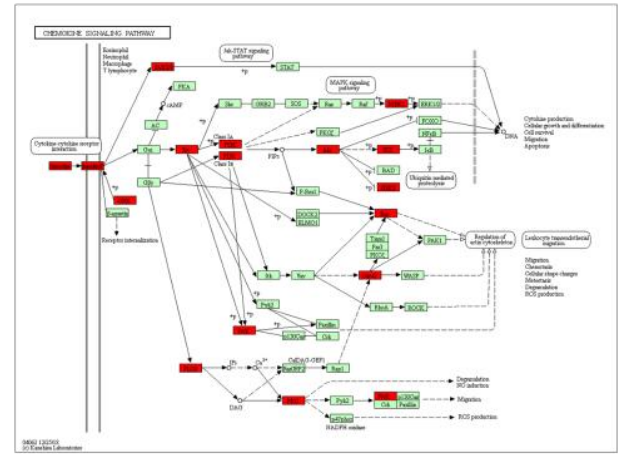

D.

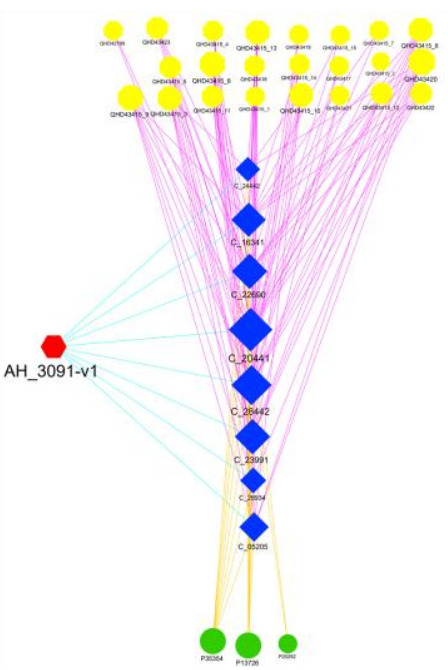

Figure 8. Immune regulatory potential of Ayurvedic herbs. A. Immunomodulatory Network: The immunoregulatory network represents the participation of protein-targets of $129 \mathrm{PEP}_{\text {cov2 }}$ in regulating the immunesystem of Homo sapiens. The outer layer representing the circular nodes depicts 19 of 21 immune-system related pathways in humans as described by KEGG database. Among 373 human protein targets of 129 PEP $_{\text {cov2 }}$, 63 were found to be involved in 19 immune pathways, arranged inside the circular layout as shown by green color circular nodes. No protein target were associated with hsa04624 and hsa04625, thereby restricting the number to 19 . B. Chemokine signaling pathway (path:hsa04062) obtained from KEGG database: The location of the mapped genes corresponding to protein targets of $\mathrm{PEP}_{\text {cov } 2}$ are highlighted in red-colored boxes in the pathway. C. Herbspecific immune regulatory network (HSIR-Network): HSIR network is the 4-component network of size 352

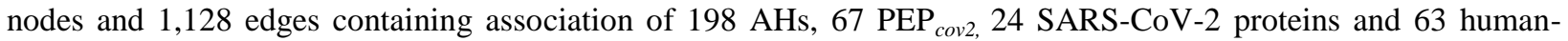
proteins. The network is limited to the protein targets involved in immune-system related pathways in humans as described by KEGG database. D. Subnetwork of HSIR-network specific to AH_3091-v1 (Ziziphus jujube): The dual-regulatory role of Ziziphus jujube in targeting SARS-CoV-2 and human immune system related proteins through 8 of its phytochemicals (C_23991, C_28934, C_22690, C_05205, C_16341, C_28442, 51025490, C_2044, and C_24442) represented by blue-colored diamonds.

The immune-regulatory network suggests that immunoregulatory effect may be conferred by carefully designed combination of phytochemicals. The combined effect of these PEPs may be 
associated with the molecular-scale rationale behind the immune-boosting capacity of Ayurvedic herbs and formulations. Among $67 \mathrm{PEP}_{\text {cov2 }}$ involved in immunoregulation (via targeting 63 human-proteins of immunoregulatory network), C_34364 and C_02937 are the top immunoregulators with 13 and 9 protein targets, respectively. Additional details of these phytochemicals may be checked by studying the phytochemical and their protein-ligand complexes for their structural and analytical properties. To derive the information of the herbs these $67 \mathrm{PEP}_{\text {cov } 2}$ (involved in immunoregulation) belongs to, the AH-PEPs network was checked that lead to the identification of association of $198 \mathrm{AHs}$ with these $67 \mathrm{PEP}_{\text {cov2. }}$. The information is added to Bi-regulatory PEP cov2 network to construct a 4-component network consisting of 198AHs, $67 \mathrm{PEP}_{\text {cov } 2}$ and their regulators from both human and SARS-CoV-2 proteins. This led to the construction of a herb-specific immune regulatory network (HSIR network), with network size of 352 nodes and 1,128 edges containing association of $198 \mathrm{AHs}, 67 \mathrm{PEP}_{\text {cov2, }} 24 \mathrm{SARS}$ CoV-2 proteins and 63 proteins from humans (Figure-8.C). Each immune-regulatory herb from the 198 AHs was prioritized on the basis of their both human and virus targeting capability. When checked for the AHs having at least 5 virus targets, 149 AHs got selected and among those the AHs who contribute maximally to the protein targets are Zea mays (AH_3081-v1) with 32 proteins being targeted by its 9 PCs, Cucurbita maxima (AH_0865-v1) with 29 proteins being targeted by its 10 PCs, Pisum sativum (AH_2237-v1) with 29 proteins being targeted by its 9 PCs, Thlaspi arvense (AH_2874-v1) with 28 protein being targeted by its 8 PCs, Calophyllum inophyllum (AH_0504-v1) with 28 protein being targeted by its 5 PCs and AH_3091-v1/v3 (Ziziphus jujube) with 27 proteins being targeted by its 2 PCs (for AH_3091-v3) and with 26 proteins being targeted by its 8 PCs (for AH_3091-v1).

Among these, Ziziphus jujube was found to target maximum number of viral proteins. It is interesting to note that the decoction of Ziziphus jujube has also been suggested in the advisory issued by Ministry of AYUSH (Ayurveda, Yoga, Unani, Siddha and Homeopathy), Government of India towards the management of COVID at its preventive and prophylactic stage 9 . The observation strengthens the credibility of the network towards suggesting potential herbs and their phytochemicals for dealing COVID-19 pandemic. The network also sheds light on the phytochemical specific targeted action of herbs for example, the targeted action of Ziziphus jujube is shown in Figure-8.D, where the mechanism of its management against COVID-19 can be attributed to its 8 phytochemicals (C_23991, C_28934, C_22690, C_05205, C_16341, 
C_28442, C_2044 and C_24442) that have targeting potential against 23 SARS-CoV-2 proteins. The plant also aids in regulation the host immune system through these 8 phytochemicals by targeting three human proteins P20292, P13726 and P35354, among these P13726 and P35354 belong to the class of FDA-approved targets. The complete interaction data used for constructing HSIR-network is given in Supplementary Table-10. The data may be checked for other herbs to decipher their phytochemical specific targeted-action in the management of COVID-19. Although the network is limited to the immune-regulatory potential of those phytochemicals having the ability to target SARS-CoV-2 proteins, the approach holds the potential to give a mechanistic understanding of therapeutic relevance of traditional herbs.

\section{Summary:}

The exceptional state of health crisis emerged due to the novel SARS-CoV-2 virus, has forced the researchers across the globe to constantly work towards searching the preventive measures as well as developing its possible cure. Ayurveda, considered as the oldest healing schema on Earth, describes thousands of herbs and their formulations for the well-being of mankind. It has always remained a great source of drugs and other lead-like molecules. To explore the therapeutic relevance of Ayurveda for combating the current situation, the network pharmacological evaluation of Ayurvedic herbs was carried out in this study. An extensive collection of the phytochemicals present in Ayurvedic-herbs and the study of their regulatory prospects form the basis of present work. To decipher the phytochemical-specific targeted action of herbs, a collection of 34,472 Ayurvedic phytochemicals (APCs) was developed from 7,258 botanical names. 292 (referred to as PEPs) of these phytochemicals were found to be similar (based on $T_{c}$ value) with 16 of 125 currently available anti-viral drugs considered in the study. Herb-wise distribution of PEPs was found to be maximally concentrated to Artemisia annua with 35 of its phytochemicals in PEPs category. When checked for the association of these 292 PEPs with SARS-CoV-2 proteins based on their binding energy value distribution, 129 (referred to as $\mathrm{PEP}_{\text {cov2) }}$ were screened-in against 24 SARS-CoV-2 proteins, thereby restricting the further analysis to $\mathrm{PEP}_{\text {cov2 }}$. The therapeutic relevance of PEPs was assessed using the information of their 621 human protein targets and 24 SARS-CoV-2 protein targets, where targeting capabilities of $62 \mathrm{PEP}_{\text {cov2 } 2}$ were identified against non-structural protein nsp6 of SARS-CoV-2. Among the 
list of $292 \mathrm{PEP}_{\text {cov2 }}, 115$ were identified with dual regulatory mode having targeting capability in both virus and its host system, thereby indicating their future implications in pharmacophorebased drug-design approaches. For example, a phytochemical C_03212 (Inophyllum B from Calophyllum inophyllum) was found to support the cardiovascular system by targeting genes involved in cardiovascular diseases, like, TLR4, PLA2G7 \& PIK3CA. The ability of this compound to target 20 SARS-CoV-2 proteins further strengthens its role in managing COVID19. The multi-regulatory role of 73 phytochemicals was highlighted for their ability to manage the complication of COVID-19 associated comorbidity, among them the effect of C_17006 (CHEMBL141117) is highly noticeable for its multi-targeting strategy. In addition to this, the high binding affinity of the compound for nsp2 protein of SARS-CoV-2 attracts attention for its ability to act as a potential lead moiety. Immunoregulatory ability of the Ayurvedic herbs was also explored and presented as a special case study. The analysis helps to decipher the role of 63 $\mathrm{PEP}_{\text {cov2 }}$ for their regulatory role on the immune system of host body where the effect is mainly via regulating chemokine and NOD-like receptor signaling pathways. C_34364 (Guanosine 5'triphosphoric acid) and C_02937 (Crotonoside) were found as top immunoregulators with ability to regulate 13 and 9 proteins of the immune system, respectively. In our study, Ziziphus jujube appeared as a potential candidate with dual-regulatory effects in SARS-CoV-2 targeting and immune-supportive role, the detailed phytochemical-special protein-targeting ability of the plant have been deciphered and presented as an example where the effect is found to be mainly through its 8 phytochemicals. Other potential herbs may also be explored for their systems-level effects and the role of multi-targeting phytochemicals can be identified via analyzing the interaction-networks generated in the study. The developed protocol provides novel insights about the complex regulatory role of traditional medicines and their target specificity in a much deeper and simpler context for managing the current global situation. This study can be considered a major attempt towards integrating the wealth of traditional practices with modern scientific approaches to meet the therapeutic demands in the current scenario.

\section{Acknowledgements:}

N.C. is grateful to the Indian Council of Medical research (ICMR) for support provided through ICMR-SRF. Authors would like to thank Dr. Arun Kumar for useful discussions. 
587 V.S. conceptualized the study and designed the research framework. N.C. contributed to data588 collection, data-integration, computational analyses. N.C. and V.S. investigated and analysed the 589 results, and prepared the manuscript.

\section{Conflict of interest:}

592 Authors declare that there is no conflict of interest regarding the publication of this work.

593

594

\section{References:}

595 1. Wang, D. et al. Clinical characteristics of 138 hospitalized patients with 2019 novel 596

597 coronavirus-infected pneumonia in Wuhan, China. Jama. 323, 1061-1069(2020).

2. Singh, V.\& Singh, V. C19-TraNet: an empirical, global index-case transmission network 598 of SARS-CoV-2. arXiv Prepr. arXiv2006.15162 (2020).

3. Pushpakom, S. et al. Drug repurposing: progress, challenges and recommendations. Nat. 601

4. Dobson, J., Whitley, R. J., Pocock, S. \& Monto, A. S. Oseltamivir treatment for influenza 602

5. Mercorelli, B., Palù, G. \& Loregian, A. Drug repurposing for viral infectious diseases: 604

605

6. Zhou, Y. et al. Network-based drug repurposing for novel coronavirus 2019-nCoV/SARS606 CoV-2. Cell Discov.6, 14 (2020).

607 7. Ren, J., Zhang, A.-H. \& Wang, X.-J. Traditional Chinese medicine for COVID-19 $608 \quad$ treatment. Pharmacol. Res.155, 104743 (2020).

609 8. Xu, J. \& Zhang, Y. Traditional Chinese Medicine treatment of COVID-19. Complement. 610

9. Ministry of AYUSH, Gov. of India. ANNEXURE-I ADVISORY FROM MINISTRY OF AYUSH FOR MEETING THE CHALLENGE ARISING OUT OF SPREAD OF CORONA VIRUS (COVID-19) IN INDIA. (2020). https://www.ayush.gov.in/docs/125.pdf

615 10. Jadhav, P., Kapoor, N., Thomas, B., Lal, H. \& Kshirsagar, N. Antiviral potential of 
selected Indian medicinal (ayurvedic) plants against herpes simplex virus 1 and 2. N. Am. J. Med. Sci.4, 641 (2012).

11. Hopkins, A. L. Network pharmacology: The next paradigm in drug discovery. Nature Chemical Biology.4, 682 (2008).

12. Choudhary, N. \& Singh, V. A census of P. longum's phytochemicals and their network pharmacological evaluation for identifying novel drug-like molecules against various diseases, with a special focus on neurological disorders. PLoS One.13, 0191006 (2018).

13. Zhang, R., Zhu, X., Bai, H. \& Ning, K. Network Pharmacology Databases for Traditional Chinese Medicine: Review and Assessment. Frontiers in Pharmacology. 10, 123 (2019).

14. Mohanraj, K. et al. IMPPAT: A curated database of Indian Medicinal Plants, Phytochemistry and Therapeutics. Sci. Rep.8, 4329 (2018).

15. Zhang, R., Yu, S., Bai, H. \& Ning, K. TCM-Mesh: the database and analytical system for network pharmacology analysis for TCM preparations. Sci. Rep.7, 1-14 (2017).

16. Zeng, X. et al. NPASS: natural product activity and species source database for natural product research, discovery and tool development. Nucleic Acids Res.46, D1217-D1222 (2018).

17. Bolton, E. E., Wang, Y., Thiessen, P. a. \& Bryant, S. H. PubChem: Integrated Platform of Small Molecules and Biological Activities. Annu. Rep. Comput. Chem.4, 217-241 (2008).

18. Gaulton, A. et al. ChEMBL: a large-scale bioactivity database for drug discovery. Nucleic Acids Res.40, D1100-D1107 (2012).

19. Djoumbou Feunang, Y. et al. ClassyFire: automated chemical classification with a comprehensive, computable taxonomy. J. Cheminform.8, 61 (2016).

20. Backman, T. W. H., Cao, Y. \& Girke, T. ChemMine tools: An online service for analyzing and clustering small molecules. Nucleic Acids Res.39, 486-491(2011).

21. Szklarczyk, D. et al. STITCH 5: Augmenting protein-chemical interaction networks with tissue and affinity data. Nucleic Acids Res.44, 380-384(2016).

22. Daina, A., Michielin, O. \& Zoete, V. SwissTargetPrediction: updated data and new features for efficient prediction of protein targets of small molecules. Nucleic Acids Res.47, W357-W364 (2019).

23. Liu, T., Lin, Y., Wen, X., Jorissen, R. N. \& Gilson, M. K. BindingDB: A web-accessible database of experimentally determined protein-ligand binding affinities. Nucleic Acids Res.35, 198-201(2007).

24. O’Boyle, N. M. et al. Open Babel: An Open chemical toolbox. J. Cheminform.3, 
650

651

652

653

654

655

656

657

658

659

660

661

662

663

664

665

666

667

668

669

670

671

672

673

674

675

676

677

678

679

680

681

682

25. Willett, P., Barnard, J. M. \& Downs, G. M. Chemical similarity searching. J. Chem. Inf. Comput. Sci.38, 983-996 (1998).

26. Choudhary, N. \& Singh, V. Insights about multi-targeting and synergistic neuromodulators in Ayurvedic herbs against epilepsy: integrated computational studies on drug-target and protein-protein interaction networks. Sci. Rep.9, 10565 (2019).

27. Piñero, J. et al. DisGeNET: A comprehensive platform integrating information on human disease-associated genes and variants. Nucleic Acids Res.45, D833-839(2017).

28. Morris, G. \& Huey, R. AutoDock4 and AutoDockTools4: Automated docking with selective receptor felxibility. J.Comput. Chem. 30, 2785-279 (2009).

29. Trott, O. \& Olson, A. J. Software news and update AutoDock Vina: Improving the speed and accuracy of docking with a new scoring function, efficient optimization, and multithreading. J. Comput. Chem.31, 455-461(2010).

30. Li, S. et al. Identification of natural compounds with antiviral activities against SARSassociated coronavirus. Antiviral Res.67, 18-23 (2005).

31. Haq, F. U. et al. Artemisia annua: trials are needed for COVID-19. Phyther. Res.2020, 1$2(2020)$.

32. Rastogi, S., Pandey, D. N. \& Singh, R. H. COVID-19 Pandemic: A pragmatic plan for Ayurveda Intervention. J. Ayurveda Integr. Med. (2020). https://doi.org/10.1016/j.jaim.2020.04.002

33. Wang, G., Tang, W. \& Bidigare, R. R. Terpenoids As Therapeutic Drugs and Pharmaceutical Agents BT - Natural Products: Drug Discovery and Therapeutic Medicine. in (eds. Zhang, L. \& Demain, A. L.) 197-227 (Humana Press, 2005). doi:10.1007/978-1-59259-976-9_9

34. De Clercq, E. \& Li, G. Approved antiviral drugs over the past 50 years. Clin. Microbiol. Rev.29, 695-747 (2016).

35. Zhao, Y., Liu, D., Proksch, P., Zhou, D. \& Lin, W. Truncateols OV, further isoprenylated cyclohexanols from the sponge-associated fungus Truncatella angustata with antiviral activities. Phytochemistry155, 61-68 (2018).

36. Zhao, Y. et al. Truncateols A-N, new isoprenylated cyclohexanols from the spongeassociated fungus Truncatella angustata with anti-H1N1 virus activities. Tetrahedron71, 2708-2718 (2015).

37. Geng, H. et al. The putative protein 6 of the severe acute respiratory syndrome-associated coronavirus: Expression and functional characterization. FEBS Lett.579, 6763-6768 
(2005).

38. Tangudu, C., Olivares, H., Netland, J., Perlman, S. \& Gallagher, T. Severe acute respiratory syndrome coronavirus protein 6 accelerates murine coronavirus infections. $J$. Virol.81, 1220-1229 (2007).

39. Yang, H., Bartlam, M. \& Rao, Z. Drug design targeting the main protease, the Achilles' heel of coronaviruses. Curr. Pharm. Des.12, 4573-4590 (2006).

40. Ramsay, R. R., Popovic-Nikolic, M. R., Nikolic, K., Uliassi, E. \& Bolognesi, M. L. A perspective on multi-target drug discovery and design for complex diseases. Clin. Transl. Med.7, 3 (2018).

41. Cheng, F., Kovács, I. A. \& Barabási, A.-L. Network-based prediction of drug combinations. Nat. Commun.10, 1197 (2019).

42. Wambier, C. G. \& Goren, A. SARS-COV-2 infection is likely to be androgen mediated. $J$. Am. Acad. Dermatol.83, 308-309(2020).

43. Zheng, Y.-Y., Ma, Y.-T., Zhang, J.-Y. \& Xie, X. COVID-19 and the cardiovascular system. Nat. Rev. Cardiol.17, 259-260 (2020).

44. Choudhary, N., Choudhary, S. \& Singh, V. Deciphering the multi-scale mechanisms of Tephrosia purpurea against polycystic ovarian syndrome (PCOS) and its major psychiatric comorbidities: studies from network-pharmacological perspective. bioRxiv 785048 (2019).

45. Jia, S.-J., Niu, P.-P., Cong, J.-Z., Zhang, B.-K. \& Zhao, M. TLR4 signaling: A potential therapeutic target in ischemic coronary artery disease. Int. Immunopharmacol.23, 54-59 (2014).

46. Zalewski, A. \& Macphee, C. Role of lipoprotein-associated phospholipase A2 in atherosclerosis: biology, epidemiology, and possible therapeutic target. Arterioscler. Thromb. Vasc. Biol.25, 923-931 (2005).

47. Durrant, T. N. \& Hers, I. PI3K inhibitors in thrombosis and cardiovascular disease. Clin. Transl. Med.9, 8 (2020).

48. Yimdjo, M. C. et al. Antimicrobial and cytotoxic agents from Calophyllum inophyllum. Phytochemistry65, 2789-2795 (2004).

49. Patil, A. D. et al. The inophyllums, novel inhibitors of HIV-1 reverse transcriptase isolated from the Malaysian tree, Calophyllum inophyllum Linn. J. Med. Chem.36, 41314138 (1993).

50. Fang, L., Karakiulakis, G. \& Roth, M. Are patients with hypertension and diabetes mellitus at increased risk for COVID-19 infection? Lancet Respir. Med.8, 21 (2020). 
716 51. Shi, Y. et al. COVID-19 infection: the perspectives on immune responses. Cell Death 717 Differ.27, 1451-1454 (2020).

718 52. Patel, J., Channon, K. M. \& McNeill, E. The downstream regulation of chemokine 719 receptor signalling: implications for atherosclerosis. Mediators Inflamm.2013, $720 \quad 459520(2013)$.

721

722 